\title{
EDITORIAL
}

\section{Glucocorticoid treatment and cardiovascular disease}

\author{
M K C Ng, D S Celermajer
}

Heart 2004;90:829-830. doi: 10.1136/hrt.2003.031492

The propensity of glucocorticoids to produce hyperglycaemia, hypertension, dyslipidaemia, and central obesity has long been a cause for concern among physicians regarding possible adverse cardiovascular events. Should heart failure be added to this list of concerns?

See end of article for authors' affiliations ......................

Correspondence to: Professor David S Celermajer, Department of Cardiology, Royal Prince Alfred Hospital

Camperdown, Sydney, NSW 2050, Australia; david.celermajer@email. cs.nsw.gov.au n 1948, the US rheumatologist Philip Hench and his associates at the Mayo Clinic first administered hydrocortisone to a patient with rheumatoid arthritis and discovered its clinical benefits. ${ }^{1}$ Two years later, Hench, together with biochemists Edward Kendall and Tadeus Reichstein, shared the Nobel Prize in Medicine. Today, glucocorticoids are among the most frequently prescribed class of anti-inflammatory medications. ${ }^{2}$ They are part of the standard treatment for a wide range of disorders which feature inflammation and/or immune activation, such as asthma, chronic obstructive pulmonary disease, hypersensitivity reactions, autoimmune diseases, and in organ transplantation. However, even early on, the euphoria generated by the discovery of corticosteroids was rapidly tempered by the realisation that clinicians were, in a sense, engaging in a Faustian pact between its impressive anti-inflammatory benefits and its potentially devastating Cushingoid side effects. ${ }^{34}$

\section{CARDIOVASCULAR EFFECTS OF GLUCOCORTICOIDS}

From a cardiovascular standpoint, the propensity of glucocorticoids to produce hyperglycaemia, hypertension, dyslipidaemia, and central obesity has long produced concern regarding possible adverse cardiovascular events. ${ }^{5}$ Glucocorticoid administration increases blood pressure in a dose dependent fashion. The mechanisms of glucocorticoid mediated hypertension are incompletely understood but appear to be principally related to increased peripheral vascular resistance rather than to mineralocorticoid receptor mediated effects of increased sodium retention and plasma volume expansion. ${ }^{6}$ Dyslipidaemia in the context of long term glucocorticoid use is characterised by increased total cholesterol, low density lipoprotein cholesterol, and triglycerides. ${ }^{2}$ Corticosteroid treatment increases the risk of glucose intolerance in patients without known diabetes and is associated with deterioration of glycaemic control in diabetic patients. ${ }^{7}$ Glucocorticoid treatment therefore contributes to the exacerbation of a cluster of cardiovascular risk factors that are central to the metabolic syndrome. However, as inflammation plays a central role in the pathogenesis in atherosclerosis, ${ }^{8}$ it is also possible that glucocorticoids may exert some anti-atherosclerotic effects. Indeed, dexamethasone has been shown to reduce atherosclerosis ${ }^{910}$ and restenosis ${ }^{11}$ in experimental animal models. Furthermore, high dose glucocorticoid treatment has been shown to protect the myocardium from acute ischaemic injury in some animal models. ${ }^{12}{ }^{13}$

Glucocorticoid activation of the mineralocorticoid receptor may also exert clinically relevant cardiovascular effects. The division of adrenal steroids into glucocorticoids and mineralocorticoids is somewhat arbitrary in that most glucocorticoids activate both of these receptors. Recent randomised clinical studies have shown that mineralocorticoid receptor blockade using spironolactone $^{14}$ or the selective aldosterone antagonist, eplerenone, ${ }^{15}$ reduces morbidity and mortality in patients with heart failure. These studies have highlighted a role for mineralocorticoid receptor activation in the pathophysiology of this condition. Mechanistic studies suggest that mineralocorticoid activation exacerbates heart failure not just by increasing sodium and fluid retention but also by promoting remodelling through fibrosis of the atria and ventricles. ${ }^{16}$ Mineralocorticoid effects of administered glucocorticoids may therefore increase the risk of heart failure.

\section{GLUCOCORTICOID TREATMENT AND CARDIOVASCULAR EVENTS}

The effect of glucocorticoids on cardiovascular events has never been well documented, mainly because no large randomised trials have been performed with oral steroid treatment that have been powered to detect differences in cardiovascular morbidity and mortality. In this context, the very careful and large observational study of Souverein and colleagues ${ }^{17}$ in this issue of Heart attempts to address the possible association between oral glucocorticoids and the risk of cardiovascular and cerebrovascular disease, in a population based case-control study. Although retrospective and observational, the authors have studied an impressive 50656 cases and an equivalent number of matched controls, and have been very careful to consider and adjust for potential confounders. They report that oral glucocorticoids appear to be associated with a substantial dose dependent increase in the risk of heart failure (adjusted odds ratio (OR) 2.66, 95\% confidence interval (CI) 2.46 to 2.87). These results are probably unsurprising, given the hypertensive effects of steroids and the deleterious effects of mineralocorticoid receptor activation. Of concern, these effects could be expected 
to unmask otherwise subclinical heart failure in at-risk subjects.

Much less impressive was the effect of glucocorticoid treatment on the risk of atherosclerosis, a mild (although significant) effect only on incident coronary artery disease (adjusted OR 1.20, 95\% CI 1.11 to 1.29) and an apparent negative association with cerebrovascular disease (adjusted OR $0.91,95 \%$ CI 0.84 to 0.99 ). This is despite the well known effects of glucocorticoids on insulin resistance, blood sugar, blood lipids, blood pressure, and obesity-all recognised risk factors for coronary artery disease. These data raise the possibility that some of these potentially pro-atherogenic influences may be attenuated by possible anti-inflammatory effects of glucocorticoids.

\section{NOVEL EFFECTS OF GLUCOCORTICOIDS AND FUTURE DIRECTIONS}

There are many aspects of steroid action that we do not yet know or are just beginning to appreciate. The classic glucocorticoid and mineralocorticoid receptors belong to a superfamily of ligand dependent nuclear transcription factors that mediate their effects by altering gene expression. ${ }^{18}$ However, in addition to these classic genomic effects, recent studies have shown that glucocorticoids also exert rapid, non-genomically mediated effects, possibly via activation of a putative membrane bound glucocorticoid receptor or by direct physicochemical interactions with cell membrane constituents. ${ }^{19}$ Emerging evidence indicates that the nongenomic effects of glucocorticoids may in part be responsible for its beneficial effects on vascular inflammation, including the inhibition of leucocyte-endothelial interactions ${ }^{20}$ and the non-transcriptional activation of endothelial nitric oxide synthase. ${ }^{21}$ Further delineation and dissection of genomic and non-genomic glucocorticoid effects will be important in facilitating more specific pharmacological targeting of steroid effects-such that its beneficial effects can be achieved with minimal adverse effects.

Despite the inherent limitations of a retrospective observational study, Souverein and colleagues ${ }^{17}$ have therefore contributed a careful and much needed analysis of the potential relation between oral glucocorticoid treatment and cardiovascular outcomes in a very large number of patients. Their principal finding, that oral glucocortoid treatment may be a risk factor for heart failure, is thought provoking and hypothesis generating, but certainly should not change clinical practice. Glucocorticoids are usually used by primary and specialist physicians only in cases of important likely benefit, because of their well known acute and more chronic adverse affects. Nevertheless, physicians should be aware of the potentially important association between glucocorticoid treatment and clinically apparent heart failure, particularly in high risk subjects with known or suspected cardiovascular disease.

\section{ACKNOWLEDGEMENTS}

Dr $\mathrm{Ng}$ is supported by the National Health and Medical Research Council of Australia.

\section{Authors' affiliations}

M K C Ng*, D S Celermajer*, Department of Medicine, University of Sydney, Australia

*Also Heart Research Institute, Sydney, Australia

\section{REFERENCES}

1 Hench PS, Kendall EC, Slocumb CH, et al. The effect of a hormone of the adrenal cortex (17-hydroxy-11-dehydrocorticosterone, compound 8E) and of pituitary adrenocorticotrophic hormone on rheumatoid arthritis. Proc Staff Meet Mayo Clin 1949;24:181-97.

2 Schacke H, Docke WD, Asadullah K. Mechanisms involved in the side effects of glucocorticoids. Pharmacol Ther 2002;96:23-43.

3 Plotz CM, Knowlton Al, Ragan C. The natural history of Cushing's syndrome. Am J Med 1952;13:597-614.

4 Plotz CM, Knowlton Al, Ragan C. Natural course of Cushing's syndrome as compared with the course of rheumatoid arthritis treated by hormones. Ann Rheum Dis 1952;11:308-9.

5 Sholter DE, Armstrong PW. Adverse effects of corticosteroids on the cardiovascular system. Can J Cardiol 2000;16:505-11

6 Kelly JJ, Mangos G, Williamson PM, et al. Cortisol and hypertension. Clin Exp Pharmacol Physiol Suppl 1998;25:S51-6.

7 Andrews RC, Walker BR. Glucocorticoids and insulin resistance: old hormones, new targets. Clin Sci (Lond) 1999;96:513-23.

8 Libby P. Inflammation in atherosclerosis. Nature 2002;420:868-74.

9 Makheja AN, Bloom S, Muesing R, et al. Anti-inflammatory drugs in experimental atherosclerosis in WHHL rabbits and inhibition by cortisone acetate. Atherosclerosis 1989;76:155-61.

10 Hayashi $\mathrm{H}$, Nomoto $\mathrm{A}$, Mutoh S, et al. Role of inflammatory response in initiation of atherosclerosis: effects of anti-inflammatory drugs on cuff-induced leukocyte accumulation and intimal thickening of rabbit carotid artery. Atherosclerosis 1991;91:107-16.

11 Villa AE, Guzman LA, Chen W, et al. Local delivery of dexamethasone for prevention of neointimal proliferation in a rat model of balloon angioplasty. J Clin Invest 1994;93:1243-9.

12 Libby P, Maroko PR, Bloor CM, et al. Reduction of experimental myocardial infarct size by corticosteroid administration. J Clin Invest 1973;52:599-607.

13 Spath JAJ, Lane DL, Lefer AM. Protective action of methylprednisolone on the myocardium during experimental myocardial ischemia in the cat. Circ Res 1974;35:44-51.

14 Pitt B, Zannad F, Remme WJ, et al. The effect of spironolactone on morbidity and mortality in patients with severe heart failure. Randomized aldactone evaluation study investigators. N Engl J Med 1999;341:709-17.

15 Pitt B, Remme W, Zannad F, et al. Eplerenone, a selective aldosterone blocker, in patients with left ventricular dysfunction after myocardial infarction. N Engl J Med 2003;348:1309-21.

16 Weber KT. Aldosterone in congestive heart failure. N Engl J Med 2001;345:1689-97

17 Souverein PC, Berard A, Van Staa TP, et al. Use of oral gucocorticoids and risk of cardiovascular and cerebrovascular disease in a population based case-control study. Heart 2004;90:859-65

18 Mangelsdorf DJ, Thummel $C$, Beato $M$, et al. The nuclear receptor superfamily: the second decade. Cell 1995;83:835-9.

19 Buttgereit F, Scheffold A. Rapid glucocorticoid effects on immune cells. Steroids 2002;67:529-34.

20 Pitzalis C, Pipitone N, Perretti M. Regulation of leukocyte-endothelial interactions by glucocorticoids. Ann NY Acad Sci 2002;966:108-18.

21 Hafezi-Moghadam A, Simoncini T, Yang E, et al. Acute cardiovascular protective effects of corticosteroids are mediated by non-transcriptional activation of endothelial nitric oxide synthase. Nat Med 2002;8:473-9. 